\title{
Development of Low-Noise Charge-Sensitive Preamplifier Based on PIPS Detector
}

\author{
Jiao Liu, Xin Huang, Song Qing, Qiao Zhang, Li He \\ Chengdu University of Technology, Chengdu, China \\ Email: 978912652@qq.com
}

How to cite this paper: Liu, J., Huang, X., Qing, S., Zhang, Q. and He, L. (2020) Development of Low-Noise Charge-Sensitive Preamplifier Based on PIPS Detector. Open Access Library Journal, 7: e6868. https://doi.org/10.4236/oalib.1106868

Received: September 27, 2020

Accepted: October 27, 2020

Published: October 30, 2020

Copyright $\odot 2020$ by author(s) and Open Access Library Inc.

This work is licensed under the Creative Commons Attribution International License (CC BY 4.0).

http://creativecommons.org/licenses/by/4.0/ (c) (i) Open Access

\begin{abstract}
In this paper, a low-noise charge-sensitive preamplifier (CSA) is designed based on passivation ion implanted planar silicon (PIPS: The Passivated Implanted Planar Silicon) detectors. This charge-sensitive preamplifier uses Junction Field-Effect Transistor (JFET) as the first stage of the amplifier. In order to maximize the signal-to-noise ratio and consider the resistance-capacitance matching problem, according to different PIPS detector junction capacitances, different numbers of junction field effect transistors are matched in parallel in the first stage. Slotting technology is used at the feedback end to reduce the influence of the parasitic capacitance of the PCB board on the feedback, and the feedback capacitor resistance is adjusted to achieve the best signal-to-noise ratio. Experimental test results show that the charge-sensitive preamplifier can achieve a signal-to-noise ratio greater than 100 at room temperature, the output signal rise time is less than $60 \mathrm{~ns}$, and the electronic equivalent capacitance noise is $19 \mathrm{eV} / \mathrm{pF}$. The charge-sensitive preamplifier is connected to the Canberra PD450-17-100AM PIPS detector, and the standard $\alpha$ source ${ }^{238} \mathrm{Pu}$ is measured in a vacuum environment. The energy resolution can reach 17keV@5499keV.
\end{abstract}

\section{Subject Areas \\ Nuclear Physics \\ Keywords \\ Charge Sensitive Preamplifier, Junction Field Effect Tube, Low Noise, High Resolution}

\section{Introduction}

The development of alpha spectrometer requires a reliable, sturdy and stable 
charged particle detector, and also requires the detector to have low noise, high resolution and good detection efficiency. In practical applications, it is known that semiconductor detectors have the best energy resolution and are suitable for the development of alpha spectrometers. After years of research and development of silicon detection technology, the Canberra Company in the United States has developed a PIPS detector with low leakage current, low noise, thin entrance window, small size, and scrubbing. However, the output signal amplitude of this type of semiconductor detector is small, and the output signal is easily affected by the junction capacitance of the detector itself and the distributed capacitance at the input of the amplifier. Therefore, in the application of high-resolution spectrometer measurement systems, charge sensitive preamplifiers are often used to process the output signal of the detector as the first step to improve the system's signal-to-noise ratio and resolution [1]. This paper designs a charge-sensitive preamplifier that uses junction field effect transistors as the first-stage amplification, mainly based on resistance capacitance feedback, and greatly reduces system noise through techniques such as trenching and special layout, and improves the signal-to-noise ratio. Tested by experimental results, the performance indicators of this CSA are far superior to general charge-sensitive preamplifiers. The detection of alpha particles by the coupled semiconductor detector greatly improves the performance of the semiconductor alpha spectrometer and has made major breakthroughs in nuclear detection technology.

\section{PIPS Detector}

\subsection{Features of PIPS Detector}

The detection device adopts the PD series PIPS detector of CANBERRA Company. The PIPS semiconductor detector is a junction semiconductor detector. It adopts ion implantation plane technology and has the advantages of small leakage current and high energy resolution. The charged particles interact in the depletion zone of the detector to generate a large number of electron-hole pairs. Their initial spatial distribution depends on the type and energy of the rays. Subsequently, these electron-hole pairs are separated and collected within a certain period of time, which depends on the geometric position of the electron-hole pairs, the electric field strength and electric field distribution in the depletion region of the detector, and the carrier migration at the operating temperature of the device Rate [2].

The energy resolution of the PIPS detector is good. The detector uses $0.5 \mathrm{um}$ thick aluminum and 1 um varnish encapsulation, which does not leak light and resists harsh environments. The thickness of Van Lishui is equivalent to the equivalent thickness of 0.6 um silicon. The energy scattering and absorption caused by alpha particles are negligible compared to the scattering and absorption caused by the air between the filter paper and the detector. Compared with the traditional gold-silicon surface barrier detector, the leakage current level is 
one order of magnitude smaller. Compared with the gas detector, it does not require high voltage, does not need to be sealed, and has the advantages of good line shape. It is the most ideal detector technology for charged particle detection [2].

\subsection{Technical Indicators of PIPS Detector}

The PIPS detector has a large operating temperature range, up to $100^{\circ} \mathrm{C}$, the lowest temperature is $-30^{\circ} \mathrm{C}$, the storage temperature needs to be below $100^{\circ} \mathrm{C}$, and the leakage current is generally about tens of $\mathrm{nA}$. The bias voltage is typically $+40 \mathrm{~V}$. The sensitive area of the PD450 detector used in the system is 450 $\mathrm{mm}^{2}$, and the thickness of the sensitive area is $100-1000 \mu \mathrm{m}$.

\section{Circuit Overall Structure Design}

This CSA circuit structure is shown as in Figure 1, is composed of three parts. In order to provide a more stable voltage to the detector, part A constitutes a high-voltage bias filter circuit. Part B is the core circuit of the charge-sensitive preamplifier, where R7 is the bias resistor of the passivation ion implanted

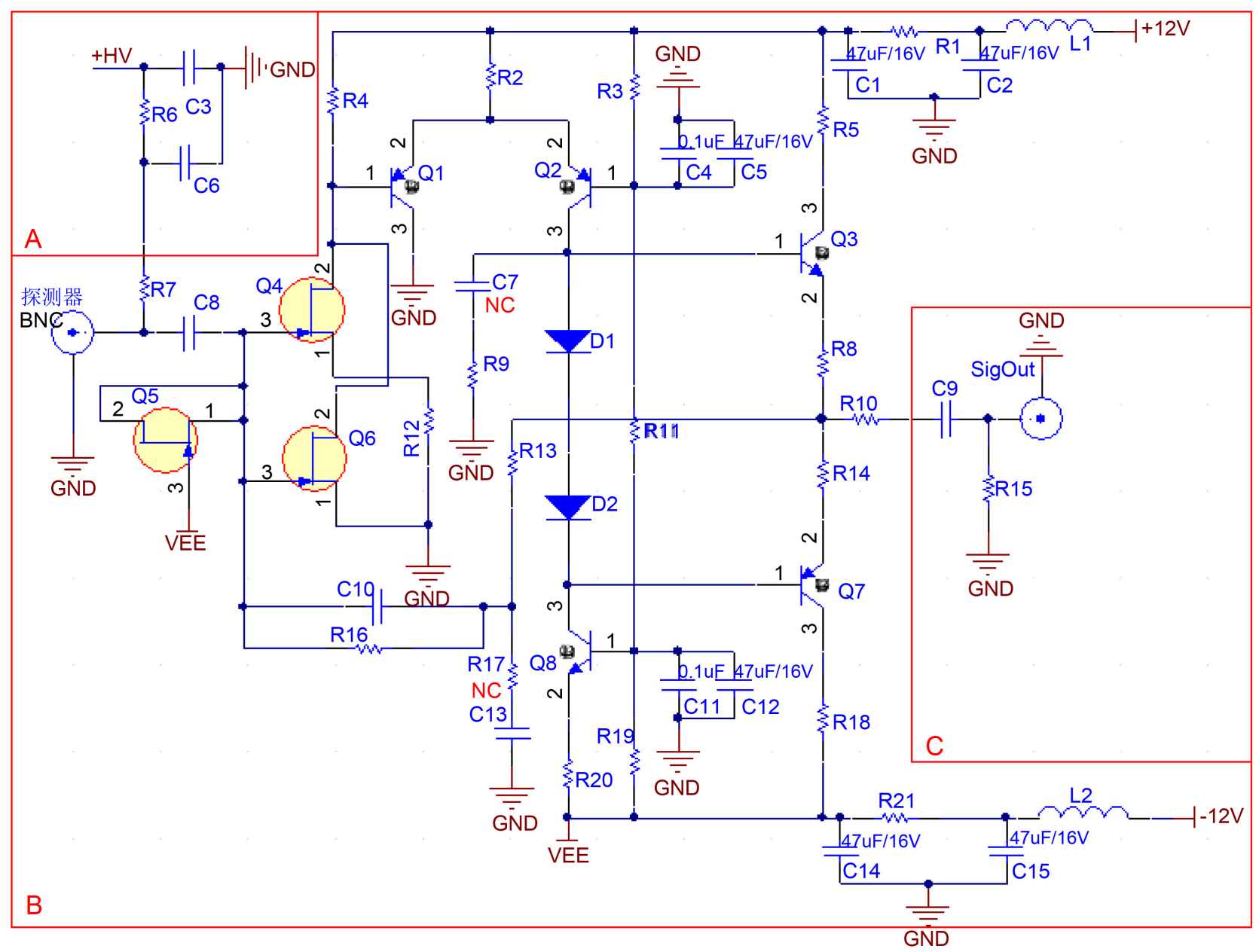

Figure 1. Circuit structure diagram. 
planar silicon PIPS detector. The shunt resistor is used to generate a voltage drop of about 0.5 volts across them [3]. And in order to avoid bringing obvious pole zeros to the output signal of the preamplifier, the bias resistance should not be too small. The AC-coupled CSA has the characteristics of simple structure, low noise, no DC offset and high counting pass rate, and is suitable for the readout circuit of the scintillation detector [4]. Therefore, this circuit adopts AC coupling mode, the output signal of the detector is blocked by AC coupling capacitor C8 and enters the input stage JFETQ4 of CSA. In order to select the CSA level $1 \mathrm{JFET}$, it is necessary to pay attention to the matching of the resistance and capacitance of the detector. The signal-to-noise ratio is highest when the input capacitance of the first-stage JFET is equal to the equivalent capacitance of the detector [5]. Therefore, in order to match the different equivalent capacitance values of different PIPS detectors, this CSA uses a corresponding number of junction FETs in parallel to increase the input capacitance of the preamplifier during the first stage input, and reduce the noise of this circuit.

Transistors Q1, Q2, and Q8 form an intermediate amplifier stage, in which Q1 and Q2 form a common set-common base amplifier circuit, which has a high input impedance; at the same time, Q1 and Q2 adopt a differential structure, and Q8 constitutes a constant current source, of which the constant current source acts as The active load of the differential amplifier circuit; Q3 and Q7 constitute the output stage of the amplifier circuit, which adopts a push-pull output structure. The diodes D1 and D2 provide the base bias voltage for Q3 and Q7 respectively, which can overcome the crossover distortion. The output stage circuit, it can reduce the output impedance of the circuit and increase the current gain.

Due to the high gain of the preamplifier circuit and the large time constant, the pulse width of the output signal is wider, which leads to the accumulation of pulses in the subsequent circuit under the condition of high counting rate, which affects the accuracy of counting. Therefore, the signal output by the preamplifier must be checked first. CR differential shaping reduces its pulse width, and then filters the signal [6]. The shaping filter circuit is shown in part C. The CR differential shaping circuit of part C selects $\mathrm{C} 9=470 \mathrm{pF}, \mathrm{R} 15=2 \mathrm{k} \Omega$, and the time constant $\tau=\mathrm{R} 15 \times \mathrm{C} 9 \approx 1 \mu \mathrm{s}$. At the same time, the differential circuit can also play a role of high-pass filtering, reducing the output signal noise.

\section{Main Performance Test and Analysis}

\subsection{Test Device}

This CSA performance test device is shown in Figure 2. The radioactive source is ${ }^{238} \mathrm{Pu}$ source, the detector is Canberra PD450-17-100AM PIPS detector, the detector bias voltage is $+35 \mathrm{~V}$, and the detector is sensitive to the charge before testing. The amplifier is placed in an aluminum metal shielded box to achieve electromagnetic shielding and reduce interference from the external environment. Because the range of alpha particles in the air is very short, about $4 \mathrm{~cm}$ to $9 \mathrm{~cm}$, and alpha particles decay quickly in the air, and there is a certain degree of 


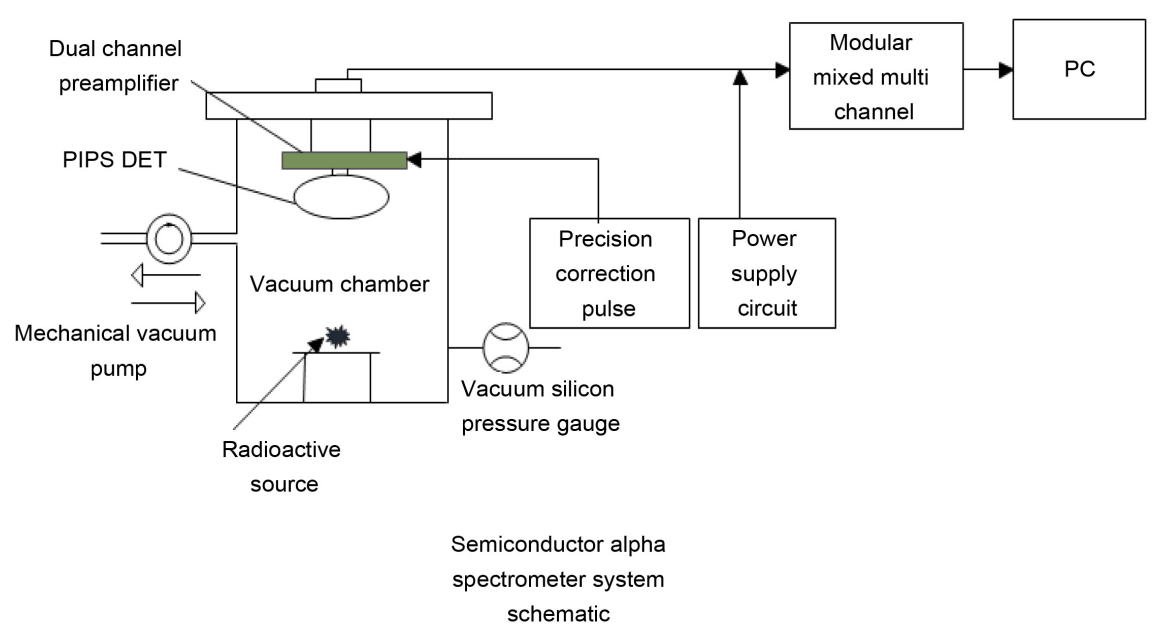

Figure 2. Diagram of test device.

loss. The spectrum measured in a vacuum environment is cleaner, and the measurement energy resolution is higher. Therefore, a mechanical vacuum pump is used to extract the air in the device in real time during the test to ensure more concentrated collection of alpha particles and more accurate experimental results.

The measurement is performed under the condition of real-time extraction of the air in the test device. To ensure the safe use of the detector, the entire device is shaded. The distance between the source and the detector is $25 \mathrm{~mm}$, the measuring window diameter of the detector is $20 \mathrm{~mm}$, and the original sample diameter on the $\alpha$ surface is $50 \mathrm{~mm}$. The test results are described below.

\subsection{Ray Output Response Test}

Connect the device according to Figure 2, and use the laboratory standard $\alpha$ source ${ }^{238} \mathrm{Pu}$ to measure the output signal as shown in Figure 3 . The output signal rise time is $40 \mathrm{~ns}$, and the circuit has excellent time response characteristics, so it can effectively reduce the number of applications in high count rate applications. Probability of signal rising edge accumulation, at room temperature, the measured signal amplitude of the ray output is about $1 \mathrm{~V}$, the baseline noise is shown in Figure 4, and the peak-to-peak value is about $10 \mathrm{mV}$. The signal-to-noise ratio reaches more than 100 at room temperature, which is much higher than the general charge-sensitive preamplifier.

\subsection{Energy Spectrum Response Test}

Couple the charge-sensitive preamplifier to the PIPS detector to test the energy spectrum response of the ${ }^{238} \mathrm{Pu}$ source: Figure 5 shows the spectrum measured by the charge-sensitive preamplifier using resistance-capacitance feedback, and its energy resolution is half-width (Full Width at Half Maxima, FWHM) = $0.32 \% @ 5499 \mathrm{keV}$, which is $17 \mathrm{keV}$, which satisfies the energy resolution of the detector itself. 


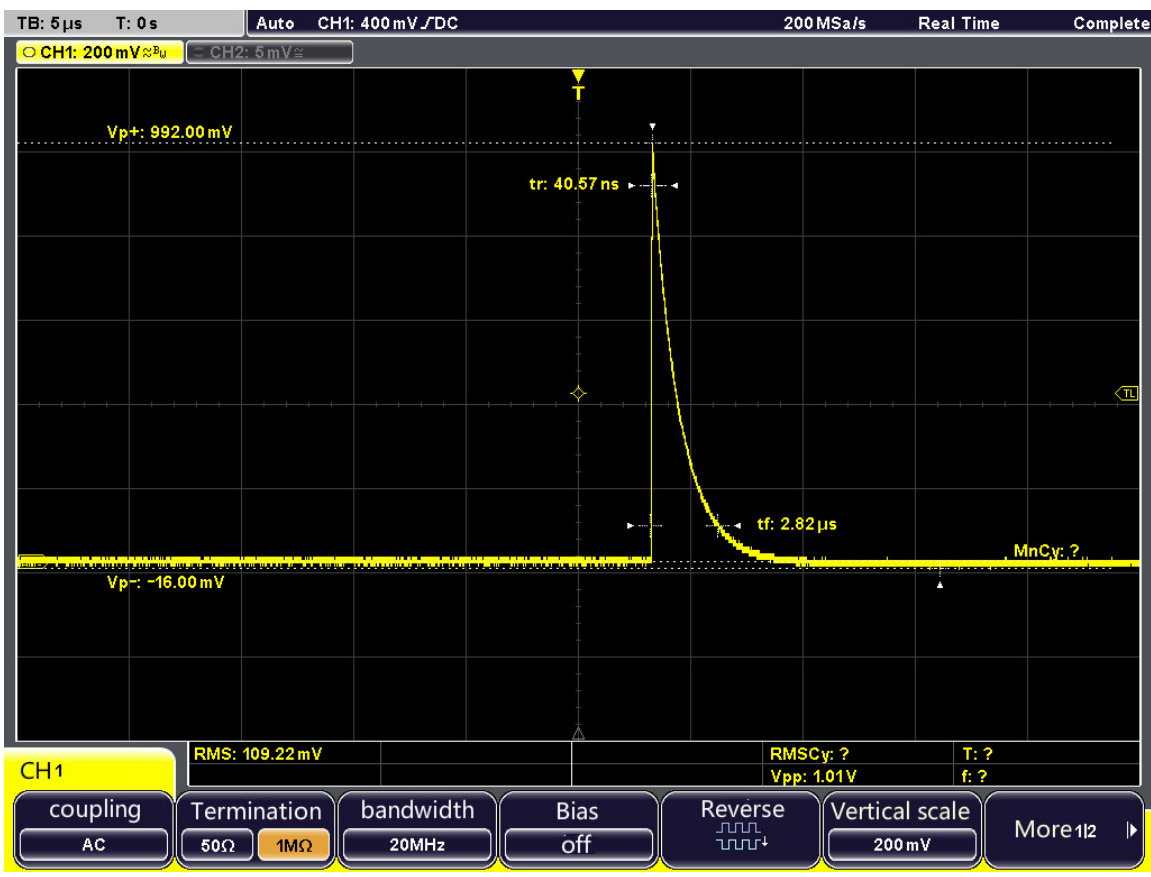

Figure 3. Charge-sensitive preamplifier test ${ }^{238} \mathrm{Pu}$ ray output signal diagram.

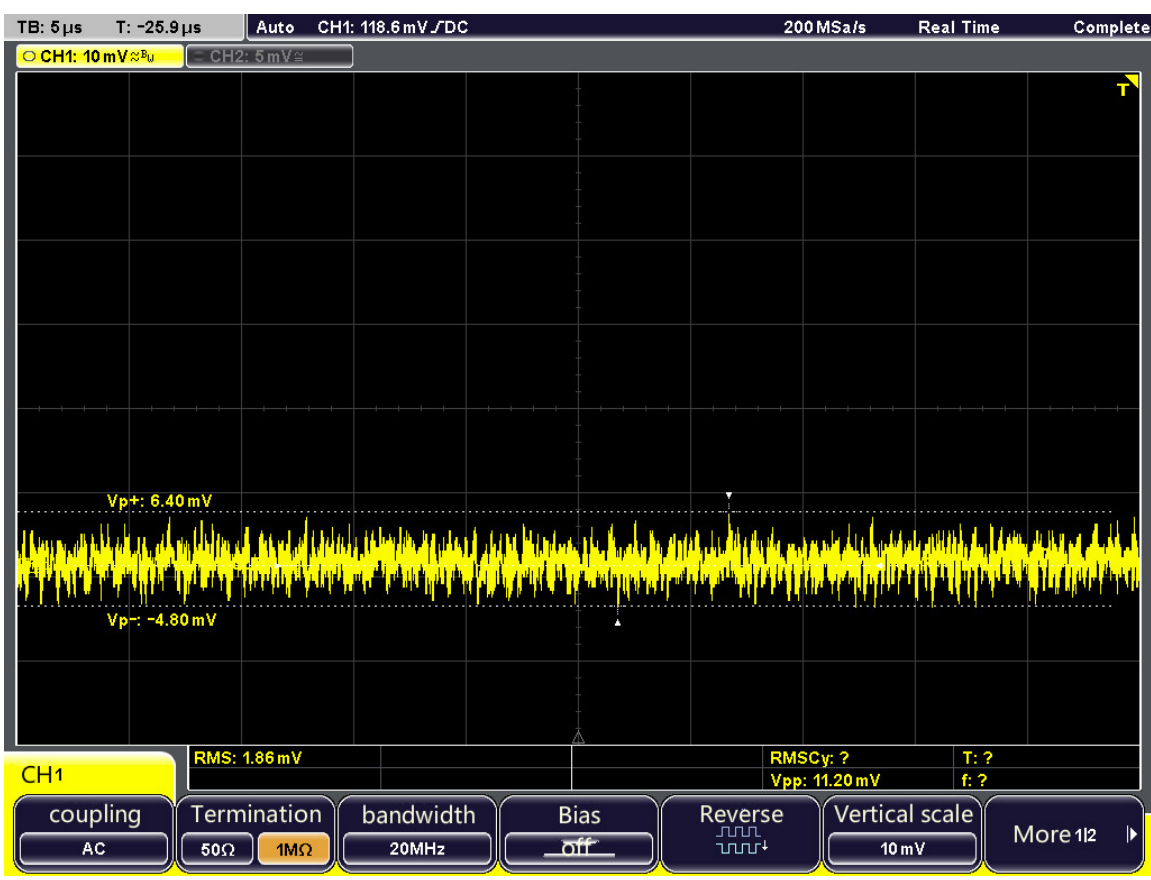

Figure 4. Baseline noise.

\section{Conclusion}

This paper is mainly based on the semiconductor PIPS detector through the adjustment of the preamplifier feedback capacitance resistance, through the technology of trenching, special layout and other techniques to design a low-noise charge-sensitive preamplifier, the experimental test data shows that it overcomes for the general traditional charge preamplifier, disadvantages of 


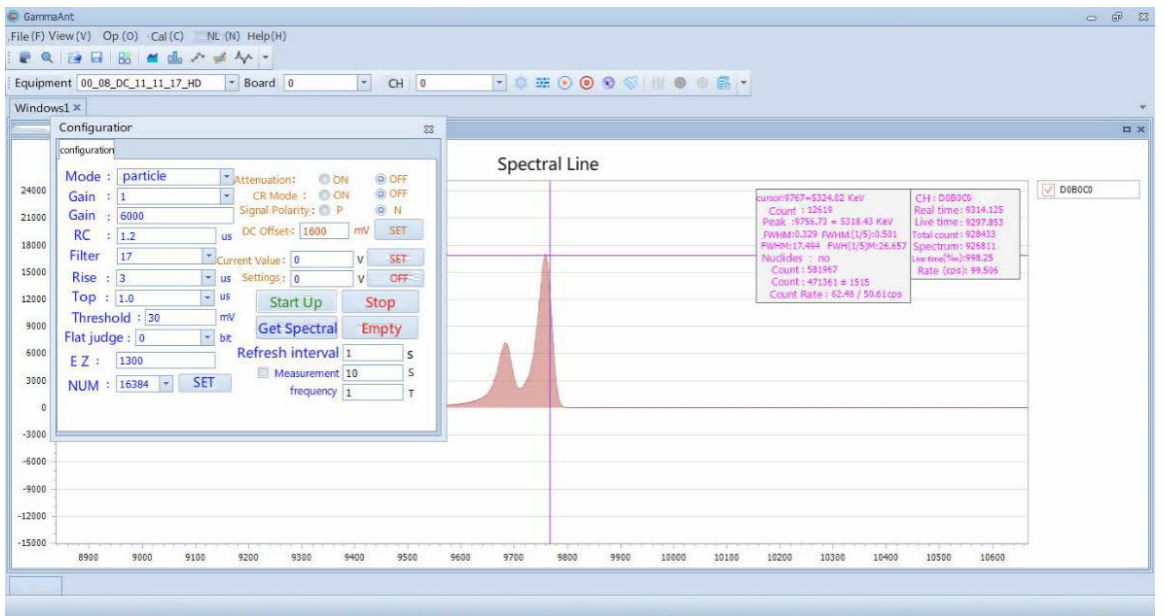

Figure $5 .{ }^{238} \mathrm{Pu}$ energy spectrum of charge-sensitive preamplifier test.

large noise, serious pulse accumulation under high count rate conditions, and low system energy resolution [7]. From the energy spectrum response test results, it can be seen that the semiconductor alpha spectrometer system using the Canberra PIPS detector and the charge preamplifier has a good energy resolution for ${ }^{238} \mathrm{Pua}$ rays $(5499 \mathrm{keV})$, which can completely replace the traditional charge preamplifier. It can be well used in high-resolution, high-count rate semiconductor alpha spectrometer systems. The next step will be the development of high-resolution semiconductor alpha spectrometers.

\section{Conflicts of Interest}

The authors declare no conflicts of interest regarding the publication of this paper.

\section{References}

[1] Wang, Y.Y., Wei, Y.K., Chen, X.L., et al. (2011) Design of Radioactive Aerosol Measurement System for Nuclear Power Plant Based on PIPS Semiconductor Detectors. Ship Science and Technology, 33, 137-139.

[2] Zou, G.J., Xia, Y., Zeng, G.Q., et al. (2016) Research on Natural Radon Thorium Progeny Detection Device Based on PIPS Detector. Computing Techniques for Geophysical and Geochemical Exploration, 38, 487-492.

[3] Yang, L., Li, S.G., Ding, Y., et al. (2017) Development of Nuclide Probe Probe Based on CdZnTe Detector. Nuclear Electronics and Detection Technology, No. 10, 1012-1016.

[4] Wang, J.J., Fan, T.M., et al. (1983) Nuclear Electronics. Volume 1, Atomic Energy Press, Beijing.

[5] Li, Y.D., Ge, L.Q., Wang, Y.Y., et al. (2017) Improved Design and Performance Study of Scintillation Probe for Portable Gamma Spectrometer. Atomic Energy Science and Technology, 51, 1471-1477.

[6] Chen, C., Hu, C.H., Zeng, G.Q., Qu, G.W., et al. (2016) Design of a New Type of a and $\beta$ Radioactivity Measurement Device. Nuclear Electronics and Detection Technology, No. 3, 241-244. 
[7] Yi, Y., Zeng, G.Q., Ge, L.Q., Hu, C.H., et al. (2019) Design of Charge-Sensitive Preamplifier Based on Resistance-Capacitance Low-Pass Feedback. Nuclear Technology, No. 9, 39-45. 